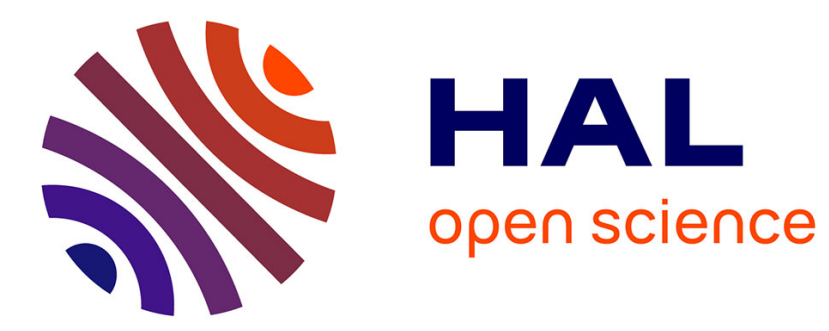

\title{
Sensorimotor control and linear visuomotor gains
}

Cécile Scotto, van Hoan Vu, Géry Casiez, Laure Fernandez

\section{To cite this version:}

Cécile Scotto, van Hoan Vu, Géry Casiez, Laure Fernandez. Sensorimotor control and linear visuomotor gains. Experimental Brain Research, 2020, 238 (9), pp.1997-2007. 10.1007/s00221-020-05856-1. hal-02919710

\section{HAL Id: hal-02919710 \\ https://hal.science/hal-02919710}

Submitted on 23 Aug 2020

HAL is a multi-disciplinary open access archive for the deposit and dissemination of scientific research documents, whether they are published or not. The documents may come from teaching and research institutions in France or abroad, or from public or private research centers.
L'archive ouverte pluridisciplinaire HAL, est destinée au dépôt et à la diffusion de documents scientifiques de niveau recherche, publiés ou non, émanant des établissements d'enseignement et de recherche français ou étrangers, des laboratoires publics ou privés. 


\title{
Authors' post-print version - Publisher version available at https://dx.doi.org/10.1007/s00221-020-05856-1
}

\section{Sensorimotor control and linear visuomotor gains}

\author{
Cécile R. Scotto https://orcid.org/0000-0002-7139-2502 1ه, Van Hoan Vu2, Géry Casiez3,4 \& Laure \\ Fernandez 2 \\ 1 Université de Poitiers; Université de Tours; Centre National de la Recherche Scientifique; \\ Centre de Recherches sur la Cognition et l'Apprentissage; Poitiers; France \\ 2 Aix Marseille Université ; Centre National de la Recherche Scientifique; Institut des Sciences \\ du Mouvement; Marseille; France \\ 3 Univ. Lille, CNRS, Centrale Lille, Inria, UMR 9189 - CRIStAL - Centre de Recherche en \\ Informatique Signal et Automatique de Lille, F-59000 Lille, France \\ 4 Institut Universitaire de France (IUF) \\ $\bowtie$ corresponding author : cecile.scotto@univ-poitiers.fr; +33 549366346
}

\begin{abstract}
In everyday life, we often use graphical interfaces where the visual space is mapped to the motor space with a visuomotor gain called the Control Display gain. One of the key objectives in the field of Human Computer Interaction is to design this Control Display gain so as to enhance users' performance . Although the Control Display gain involved in Operating Systems has been found to improve users' pointing performance, the reasons for this improvement have not yet been fully elucidated, especially because the Control Display gains on Operating Systems are both non-constant and non-linear. Here we tested non-constant but linear velocity-based Control Display gains in order to determine which parameters were responsible for pointing performance changes based on analyses of the movement kinematics. Using a Fitt's paradigm, Constantains of 1 and 3 were compared with a linearly Increasing Gain (i.e., the Control Display gain increase with the motor velocity) and a Decreasing Gain (i.e., the Control Display gain decrease with the motor velocity). Three movements with various Indexes of Difficulty (ID) were tested (ID3, ID5 and ID7). The Increasing Gain was expected to increase the velocity of the initial impulse phase and decrease that of the correction phase, thus decreasing the Movement Time (MT); and the contrary in the case of the Decreasing Gain,. Although the Decreasing Gain increased MT at ID3, the Increasing Gain was found to be less efficient than the Constant Gain of 3 , probably because a non-constant gain between the motion and its visual consequences disrupted the sensorimotor control. In addition, the kinematic analyses of the movements suggested that the motion profile was planned by the central nervous system based on the visuomotor gain at maximum motor velocity, as common features were observed between the Constant Gain of 1 and the Decreasing Gain, and between the Constant Gain of 3 and the Increasing Gain. By contrast, the amplitude of the velocity profile seemed to be specific to each particular visuomotor mapping process.
\end{abstract}

Keywords

Fitts' law $\cdot$ Pointing movement $\cdot$ Speed/accuracy trade-off $\cdot$ Control Display gain

Acknowledgements This work was funded by the ANR 'TurboTouch' project (ANR-14- CE240009). We thank Sébastien Poulmane for his assistance in developing the experimental application and Stephen Mari for his help in testing the participants in the pilot study. 
Introduction

The everyday actions we carry out in our environment usually involve a visuomotor gain which is constant and equal to one. For instance, if we reach an object by performing an arm movement, what we see about the action (in visual space) corresponds to what we are doing (in motor space). Interactions of this kind are called direct interactions. When using graphical user interfaces with two separate spaces such as laptops, however, we no longer have a one-to-one control of the environment. In these indirect configurations, the visual space (e.g., the screen) is mapped to the motor space (e.g., the trackpad) with a visuomotor gain called the Control Display (CD) gain, and generally expressed in terms of visual and motor velocities (Casiez et al. 2008). In this case, the velocity of the visual cursor corresponds to that of the end-effector multiplied by the CD gain. One of the key points in Human Computer Interaction (HCI) is to design $\mathrm{CD}$ gains enhancing users' performance on these indirect interfaces.

Recent findings on sensorimotor control have been used to assess the effectiveness of the CD gains which occur when subjects are pointing to graphical elements (e.g., icons, buttons) on these interfaces. On these lines, Fitts' law (Fitts 1954; Fitts and Peterson 1964) has been shown to predict these pointing performance: The Movement Time (MT) increases linearly depending on the Index of Difficulty (ID) of the task. The ID takes both the Width (W) and the Distance (D) to the target into account as follows: $M T=a+b \log _{2}(2 \mathrm{D} / \mathrm{W}), \mathrm{ID}=\log _{2}(2 \mathrm{D} / \mathrm{W})$, where $\mathrm{a}$ and $\mathrm{b}$ are empirical constants (Fitts 1954). This law, which was originally intended to account for direct interactions, also holds true when the CD gain differs from 1 (Card et al. 1978; MacKenzie 1992; Bohan et al. 2010). In addition, user's performance of movements on graphical interfaces can be enhanced beyond Fitts' law as graphical interfaces enable the movement to be virtual via specific physical constraints (see Balakrishnan 2004). On the one hand, pointing movements can be facilitated via tactile stimuli such as friction. For instance, the vibration of a surface creates an air film which reduces its coefficient of friction (i.e., 'squeeze film effect') and enhances pointing performance( (Casiez et al. 2011; Levesque et al. 2011). On the other hand, visual parameters were shown to enhance performance through i) movement path feedbacks (e.g., Boyle et al. 2012), ii) CD gain profiles (e.g., Blanch et al. 2004). The latter methods have been extensively tested with constant CD gains where the size of the visual screen and/or the physical size of the motor space were varied. The results obtained have often shown that MTs increased with low constant $\mathrm{CD}$ gains as well as with high constant $\mathrm{CD}$ gains, but the optimal performance was obtained at medium gains (Accot and Zhai 2001; Casiez et al. 2008; Kovacs et al. 2008; Bohan et al. 2010). While increasing the $\mathrm{CD}$ gain reduces $\mathrm{D}$ artificially, it also reduces $\mathrm{W}$, which could be detrimental to the subjects' performance. A discrete pointing movement is composed of an initial impulse phase performed at high speed, followed by a low-speed correction phase, during which visual and kinaesthetic feedback are used to reach the target accurately (Woodworth 1899; Meyer et al. 1982; Elliott et al. 2001). As high $\mathrm{CD}$ gains do not make it possible to slow down the movement during the correction phase, they may disturb the fine motor control processes. The HCI community have developed several means of overcoming these limitations by increasing the target size, shrinking the distance, or both (see Balakrishnan 2004). For instance, the non-linear mapping between the hand and cursor positions used in several studies (Fernandez and Bootsma 2008; Brenner and Smeets 2011) may have enhanced subjects' MT performance beyond the predictions of Fitts' law. Therefore, these authors showed that the reciprocal pointing (i.e., back-and-forth movement between targets) can be way faster with this visuomotor mapping which increased the $\mathrm{CD}$ gain between the targets and decreased it in the vicinity of the targets: . However, non-linear mappings based on positions are restrictive as they are semantically oriented toward the aim to achieve: The position of the target position has to be known in advance in the equation design which would not be applicable in a more ecological task where future target 
locations are unknown. Other means of pointing facilitation were subsequently developed based on velocity rather than position, with which the initial impulse phase could be performed at high speed and the correction phase at low speed. Computer operating systems based on this dynamic mapping between the visual and motor velocities (e.g., Windows, macOS and Xorg) have been found to improve users' pointing performance by shortening the MTs more than constant CD gains (Casiez et al. 2008; Casiez and Roussel 2011). However, the reasons for this improvement have not yet been completely elucidated, in particular because the gains prescribed were non-constant and non-linear. In addition, in the study by Casiez and Roussel (2011), the ID tested were high (i.e., 7 to 10.2 bits) and the comparative CD gains were low (to prevent mouse clutching). Here we assessed further how pointing performance could be improved by varying the $\mathrm{CD}$ gains in the case of high IDs as well as low IDs. For this purpose, nonconstant but linear velocity-based CD gains were used in order to determine more closely the reasons for kinematic changes in pointing movements. The results of pointing with a constant $\mathrm{CD}$ gain were compared with those of pointing movements with a linearly Increasing CD Gain $(I G)$ : The faster the effector, the faster the visual cursor moved. In order to investigate more closely how sensorimotor control with non-constant visuomotor mapping works and push this system beyond its limits, we also tested the opposite of IG: The faster the effector, the slower the visual cursor moved (Decreasing Gain: DG). The subjects' pointing performance was expected to be higher (i.e., lower MT) with IG and lower with DG in comparison with constant CD gains. More specifically, IG was expected to increase the velocity of the initial impulse phase and decrease the velocity of the correction phase, thus improving the pointing performance; and the contrary in the case of DG. A discrete pointing task was used to assess the influence of these $\mathrm{CD}$ gains on each of the two motor components: the impulse phase and the corrective phase, instead of a reciprocal pointing which does not allow to easily isolate these two processes because it is a continuous task.

\section{$2 \quad$ Methods}

\section{Participants}

Fifteen self-reported right-handed participants ( 9 men and 6 women, mean age $\pm \mathrm{SE}=27.6 \pm$ 1.0 years) gave their written informed consent prior to the study, in accordance with the 1964 Declaration of Helsinki. All the participants were right-handed and declared that they had normal or corrected-to-normal vision and no neurological or sensorimotor disorders.

\section{Apparatus}

A Fitts' task was performed on a MacBook Pro Retina (OS X 10.12 Sierra 2.9 Ghz Core i5) with a screen 13.3 inches in size (1280 x 1024 pixels, $123 \mathrm{DPI})$ refreshed at a rate of $60 \mathrm{~Hz}$. This laptop was composed of a $10.4 \mathrm{~cm} \times 7.5 \mathrm{~cm}$ trackpad with a resolution of $400 \mathrm{CPI}$, sampling at a rate of 125 $\mathrm{Hz}$. Instructions, stimuli and the data recorded from the pointing device were processed using a custom built application written in C++ using Qt and Libpointing to obtain raw input data from the pointing devices and apply the CD gain (Casiez and Roussel 2011). The application computed the visual cursor's position at $125 \mathrm{~Hz}$ based on the $\mathrm{CD}$ gain required, taking the finger speed on the trackpad computed from the absolute finger position signaled by the trackpad.

\section{Procedure}

Participants were sitting comfortably in an illuminated room in a chair with an adjustable height, facing the laptop, which they positioned themselves to facilitate the arm pointing movement. The task consisted of performing a horizontal pointing movement from left to right. The pointing movement was 
not constrained and mainly involved mobilization of the wrist. Participants were asked to point at a visual target by displacing the visual cursor $(0.2 \mathrm{~mm}$ in width; i.e., 1 pixel $)$ by sliding their right finger on the trackpad (Fig.1a). The visual target was placed $8 \mathrm{~cm}$ to the right from the starting position, and its width was either $2 \mathrm{~cm}$ ( 96 pixels), $0.5 \mathrm{~cm}$ (24 pixels) or $0.125 \mathrm{~cm}$ (6 pixels), corresponding to an ID of 3, 5 and 7 bits, respectively (Fitts 1954). The distance to the target and the location of the starting position were chosen so that it would not be possible for the finger to clutch or touch the trackpad boundaries.

In each trial, participants were instructed to explore the trackpad with their finger to find the starting position on the left of the trackpad. When this position was reached (with a tolerance of $2 \mathrm{~mm}$ ), the word "calibration" was displayed on the screen. The trial was launched when the finger stayed in this specific location for $0.5 \mathrm{~s}$. It started with the simultaneous appearance of the cursor and the visual target. Participants could then start the pointing movement whenever they were ready (Fig. 1a). They were instructed to point at the visual target as fast and as accurately as possible by making a smooth, continuous movement on the trackpad, avoiding stopping before or after the target. After a static period of $0.5 \mathrm{~s}$ on the target, the visual stimuli disappeared and the trial ended.

During the task, the CD gain between the finger and the visual cursor was manipulated. Two constant CD gains and two non-constant (but linear) CD gains were used for this purpose (Fig. 1b). The Increasing Gain $(I G)$ corresponded to a gain ranging from 1 at the motor velocity of $0 \mathrm{~cm}$.s-1 to 3 at the motor velocity of $10 \mathrm{~cm}$.s-1 or more. A pre-test was conducted in order to determine this maximum gain and the associated motor speed at which it could be reached. Five constant CD gains ( 1 to 5) were tested. This pre-test showed that the constant gain of 3 was associated with the lowest MT value and the lowest variability at all the IDs tested. In addition, we decided that this gain of 3 should be reached at a motor velocity of $10 \mathrm{~cm} . \mathrm{s}-1$ in the IG condition because it corresponded to a reachable speed whatever the ID tested. The Decreasing Gain ( $D G$ ) condition corresponded to the opposite of the IG condition: The gain value at a motor velocity of $0 \mathrm{~cm} . \mathrm{s}-1$ was 3 and decreased to 1 at a motor velocity of $10 \mathrm{~cm} . \mathrm{s}-1$ or more. IG and DG were compared at Constant Gains of 1 (CGI) and 3 (CG3), corresponding to their minimum and maximum gain values. Figure $1 \mathrm{~b}$ depicts these prescribed CD gains (CG1, CG3, IG and DG) relative to the motor velocity. For each $\mathrm{CD}$ gain, the corresponding visual velocity of the cursor relative to the motor velocity of the finger is presented in Figure 1c.

The experiment was composed of twelve blocks consisting of 30 successive trials with each of the CD gains (CG1, CG3, IG and DG) and IDs (3, 5 and 7 bits). Blocks were presented in a pseudorandom counterbalanced order. The 3 different IDs of a given $\mathrm{CD}$ gain were thus not presented successively, and half of the participants ran the blocks in the reverse order to avoid learning effects. Before the experiment, a training session was run consisting of 3 trials with each block in order to familiarize participants with the task and the instructions.

\section{b}



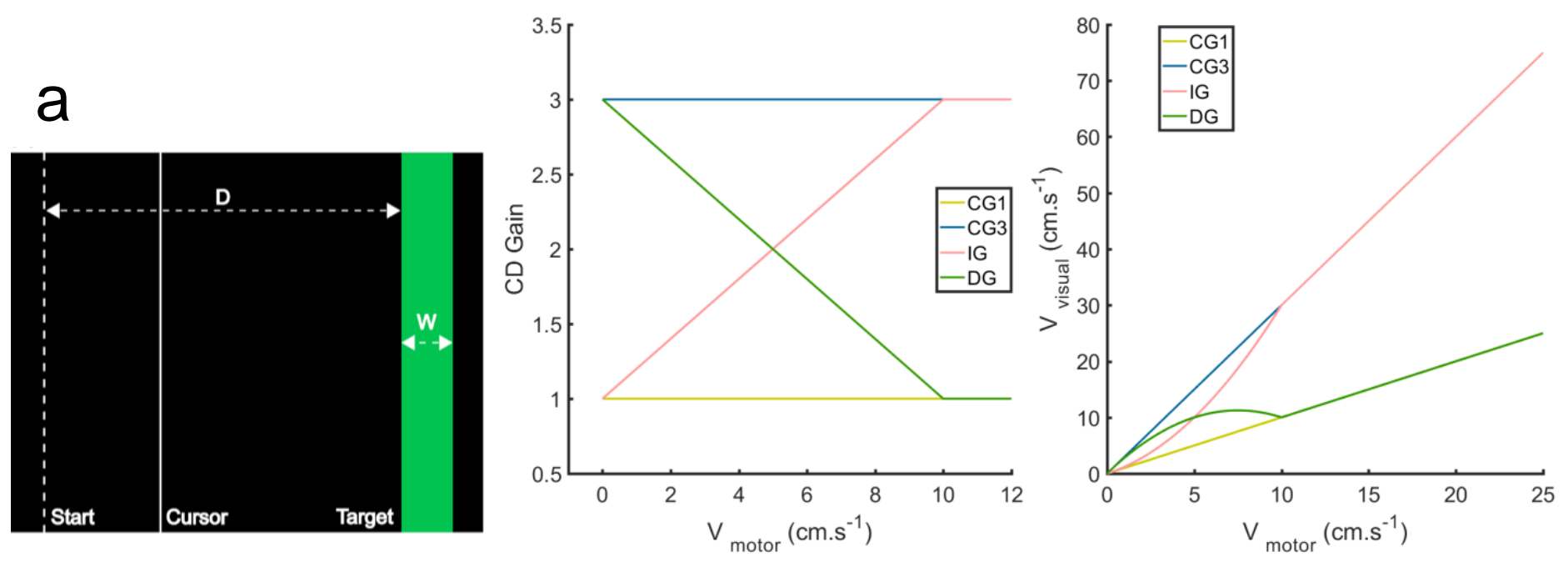

Figure 1: a) Fitt's paradigm. Participants had to move the visual cursor from the starting position to the target (width $W$ ) positioned at a distance $D$ of $8 \mathrm{~cm}$. b) Control Display (CD) gains as a function of the motor velocity (V motor). c) Predicted Visual Velocity ( $\mathrm{V}_{\text {visual }}$ ) of the cursor relative to the motor Control Display (CD) gains as a function of the Motor Velocity (Vmotor). CG1: Constant Gain of 1; CG3: Constant Gain of 3; IG: Increasing Gain; DG: Decreasing Gain.

\section{Data Processing}

Position data transmitted from the visual cursor and the trackpad were low-pass filtered with a dual-pass, no-lag Butterworth filter (cut-off frequency $10 \mathrm{~Hz}$; order 2). Data were then derivated to compute the visual and motor velocities mainly used to determine the MT of the pointing movement. The MT corresponded to the time elapsing between movement onset and offset, and is classically determined from the motor velocity (i.e., above and below 5\% of motor Peak Velocity). However, here the experimental paradigm constrained the motor velocity with the manipulation of the CD gain. The participants could move their finger slowly to reach the target quickly on the screen. Therefore, using the classical method of computation would have resulted, for instance, in an artificially longer MT with CG1 than with CG3, as the motor Peak Velocity (PV) was obviously lower in the former case. Instead, we used a fixed threshold based on visual velocity and which was coherent with task instructions which were defined in the visual space (i.e., 'point at the visual target as fast as possible [...]'). The threshold was fixed here at $5 \%$ of the median PV of all the data recorded, whatever the ID or CD gain (i.e., $5 \%$ of $19.5 \mathrm{~cm} . \mathrm{s}-1=0.975 \mathrm{~cm} . \mathrm{s}-1)$. MT therefore corresponded to the time elapsing between movement onset (i.e., Vvisual $>0.975 \mathrm{~cm} . \mathrm{s}-1$ ) and movement offset (i.e., Vvisual $<0.975 \mathrm{~cm} . \mathrm{s}-1$ and cursor position inside the target). The average MTs recorded under the various experimental conditions were compared, and a linear regression was also performed between MT and ID to test whether Fitts' law held with all the CD gains. These fittings were performed with the subjects MT's means. The pointing performance was also assessed through the endpoint variability (i.e., the standard error of the visual cursor positions in the target). Since the pointing performance was found to differ between CD gains, we investigated which motor modifications were responsible for these changes. Therefore, motor kinematic parameters were computed, such as the motor PV and amplitude (the distance covered from onset to end of the movement). We also determined whether the primary motor command and/or the online control differed between CD gains. The initial phase of movements has been reported to depend on the initial motor planning (Woodworth 1899; Meyer et al. 1982). The differences in this primary planning phase were then assessed by analyzing the distance at which the first sub-movement occurred (when the acceleration first crossed zero; Dfirstmotor; see Fradet et al. 2008) and the acceleration time (AT, the time elapsing between the movement onset and PV). In addition, the deceleration time (DT, the time elapsing between 
PV and the end of the movement) was computed as it would be associated with the correction phase composed of online corrections (Elliott et al. 2010).

The first 10 trials were not analyzed in order to prevent the occurrence of post-effects resulting from CD gain changes, as up to 10 repetitions are necessary to adapt to visuomotor modifications (Rossetti et al. 1993; Krakauer et al. 2000; Seidler et al. 2001). Statistical comparisons were then conducted on the means of the results obtained in the last 20 trials by performing repeated-measures ANOVAs using 4 CD gains (CG1, CG3, IG and DG) x 3 ID (3, 5 and 7 bits) as factors. The results were summarized in Table 1. Post-hoc tests (Newman-Keuls tests) were performed when necessary, and the level of significance was set at .05 in all the statistical analyses.

\section{Results}

Overall, the CD gain modulated the pointing movements' kinematics in both the motor and visual spaces. As shown in Figure 2, a clear-cut difference in the velocity pattern was observed between IDs. As classically established, the lower the ID was (ID3), the more symmetrical the velocity profile became (MacKenzie et al. 1987; Elliott et al. 2001). In the motor space, differences were observed between $C D$ gains, which decreased with the increase of IDs, showing a more stereotyped velocity profile at ID7. The fact that the velocity profiles also differed between CD gains in the visual space suggests that the participants did not adapt their movements in the motor space to reach a standard velocity profile in the visual space. Quantitative analyses of subjects' pointing performance and kinematic parameters are presented below. 


\section{MOTOR space}
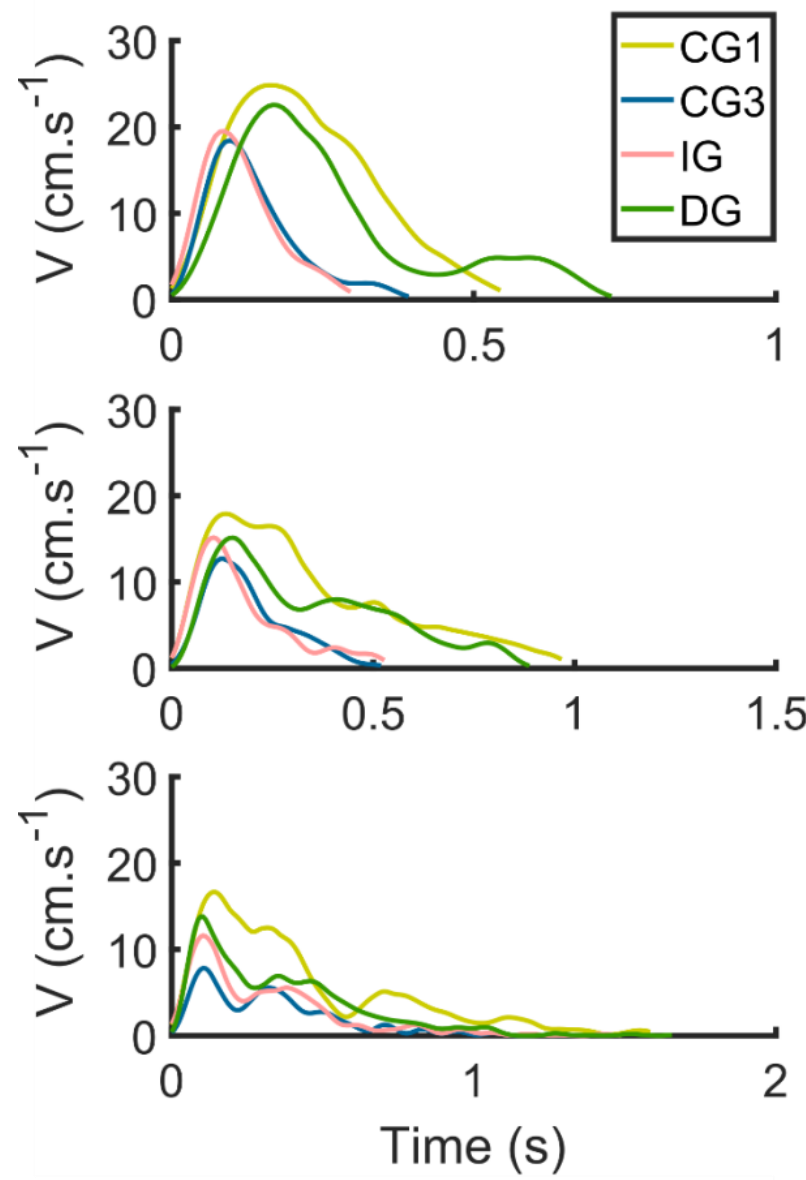

VISUAL space
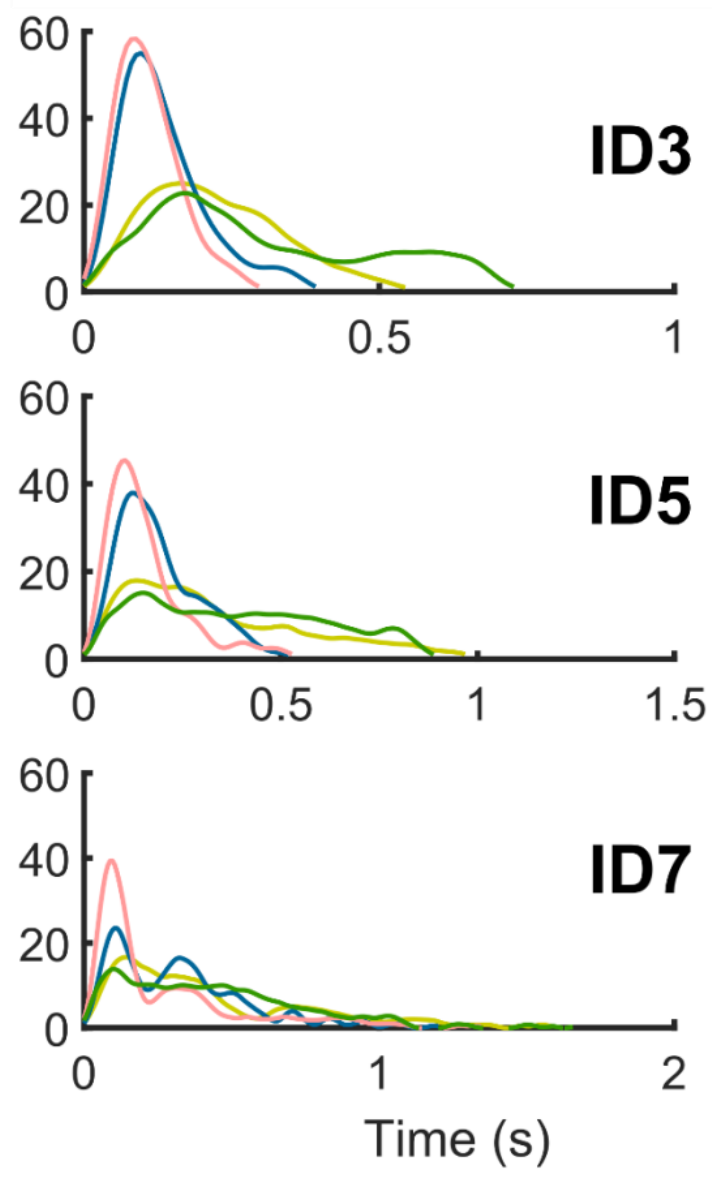

Figure 2: Velocity profiles of representative trials performed by one participant in the motor and visual spaces versus the Index of Difficulty (ID) and the CD gain. CG1: Constant Gain of 1, CG3: Constant Gain of 3; IG: Increasing Gain; DG: Decreasing Gain. $\mathrm{X}$ and $\mathrm{Y}$ axes differed depending on the ID and space.

\section{Pointing performance}

The pointing performance studied via the MTs varied in terms of the ID and CD gains. The analysis of MT revealed a main effect of ID , CD gain as well as an interaction (Table 1). Overall, MT increased with ID with all the CD gains tested (Fig. 3a). Post-hoc analysis of the interaction showed that at ID3, DG induced the highest MTs $(0.80 \pm 0.02 \mathrm{~s}$; mean \pm SE; $p<.05$ with CG1 and $\mathrm{p}<.001$ with CG3 and IG), followed by CG1 $(0.71 \pm 0.03 \mathrm{~s} ; \mathrm{p}<.01$ with CG3 and $\mathrm{p}<.001$ with IG) and by CG3 (0.56 \pm $0.05 \mathrm{~s})$ and IG $(0.55 \pm 0.04 \mathrm{~s})$, with which the fastest movement was performed (CG3 did not differ here from IG, p=0.44). At ID5, CG1 seemed as slow as DG and CG3 as fast as IG (non-significant comparisons). Lastly, at ID7, CG3 was associated with the fastest pointing performance ( $\mathrm{p}<.001$ with CG1; $\mathrm{p}<.05$ with IG and $\mathrm{p}<.01$ with DG). 
Table 1: ANOVA's result of MT (Movement Time), Endpoint variability (visual space), PX mosex $_{\text {. }}$ (Peak Velocity), Amotec (Amplitude of the pointing), rDfirstmose (Distance of the First submovement), AT (Acceleration Time) and DT (Deceleration Time)

\begin{tabular}{|c|c|c|c|c|c|c|c|}
\hline & $M T$ & $\begin{array}{c}\text { Endpeint } \\
\text { variabilito }\end{array}$ & $P V_{\text {mots }}$ & Aroster & rDfixstmentex & $A T$ & $D T$ \\
\hline$I D$ & $\begin{array}{c}F_{R 22 n}=177,0 \\
p<001\end{array}$ & $\begin{array}{c}F_{R 22 n}=115.4 \\
p<001\end{array}$ & $\begin{array}{c}F_{a 22 n}=62.9 \\
p<001\end{array}$ & $\begin{array}{c}\mathrm{F}_{(2.2 n}=1.0 \\
\mathrm{p}=.38\end{array}$ & $\begin{array}{c}F_{C 2.2 n}=\$ 2.4 \\
p<.001\end{array}$ & $\begin{array}{c}F_{c 2.2 n}=9.6 \\
p<.001\end{array}$ & $\begin{array}{c}F_{C .2 n}=158.0 \\
p<.001\end{array}$ \\
\hline$C D$ gain & $\begin{array}{c}F_{a, 421}=22.7 \\
p<001\end{array}$ & $\begin{array}{c}F_{a, 42}=21.3 \\
p<001\end{array}$ & $\begin{array}{c}\mathrm{F}_{a .42)}=102.3 \\
\mathrm{p}<001\end{array}$ & $\begin{array}{c}\mathrm{F}_{(3,42)}=564.4 \\
\mathrm{p}<001\end{array}$ & $\begin{array}{c}\mathrm{F}_{0,42}=S .1 \\
\mathbf{p}<, 01\end{array}$ & $\begin{array}{c}\mathrm{F}_{a, 421}=19.4 \\
\mathbf{p}<\mathbf{0 0 1}\end{array}$ & $\begin{array}{c}\mathrm{F}_{a, 4 y_{1}}=16.9 \\
\mathbf{p}<\mathbf{0 0 1}\end{array}$ \\
\hline ID $x$ CD gain & $\begin{array}{c}F_{(6.14)}=3.2 \\
p<01\end{array}$ & $\begin{array}{c}\mathrm{F}_{(6,14)}=9.2 \\
\mathrm{p}<001\end{array}$ & $\begin{array}{c}\mathrm{F}_{(6.14)}=2.3 \\
\mathbf{p}<.05\end{array}$ & $\begin{array}{c}F_{(s .44)}=72.8 \\
p<.001\end{array}$ & $\begin{array}{c}F_{(6.58)}=2.8 \\
p<.05\end{array}$ & $\begin{array}{c}F_{(6: t)}=1.4 \\
p=23\end{array}$ & $\begin{array}{c}F_{(6: 4)}=3.9 \\
p<01\end{array}$ \\
\hline
\end{tabular}

We then examined whether the MT increased linearly with ID, as predicted by Fitts' law. Linear regressions showed significant correlations with all the $C D$ gains $\left(C G 1: R^{2}=0.66 p<.001 ; C G 3: R^{2}=0.73\right.$ $\mathrm{p}<.001 ;$ IG: $\mathrm{R}^{2}=0.72 \mathrm{p}<.001 ; \mathrm{DG}: \mathrm{R}^{2}=0.72 \mathrm{p}<.001$ ): Fitts' law held true in all the cases investigated.
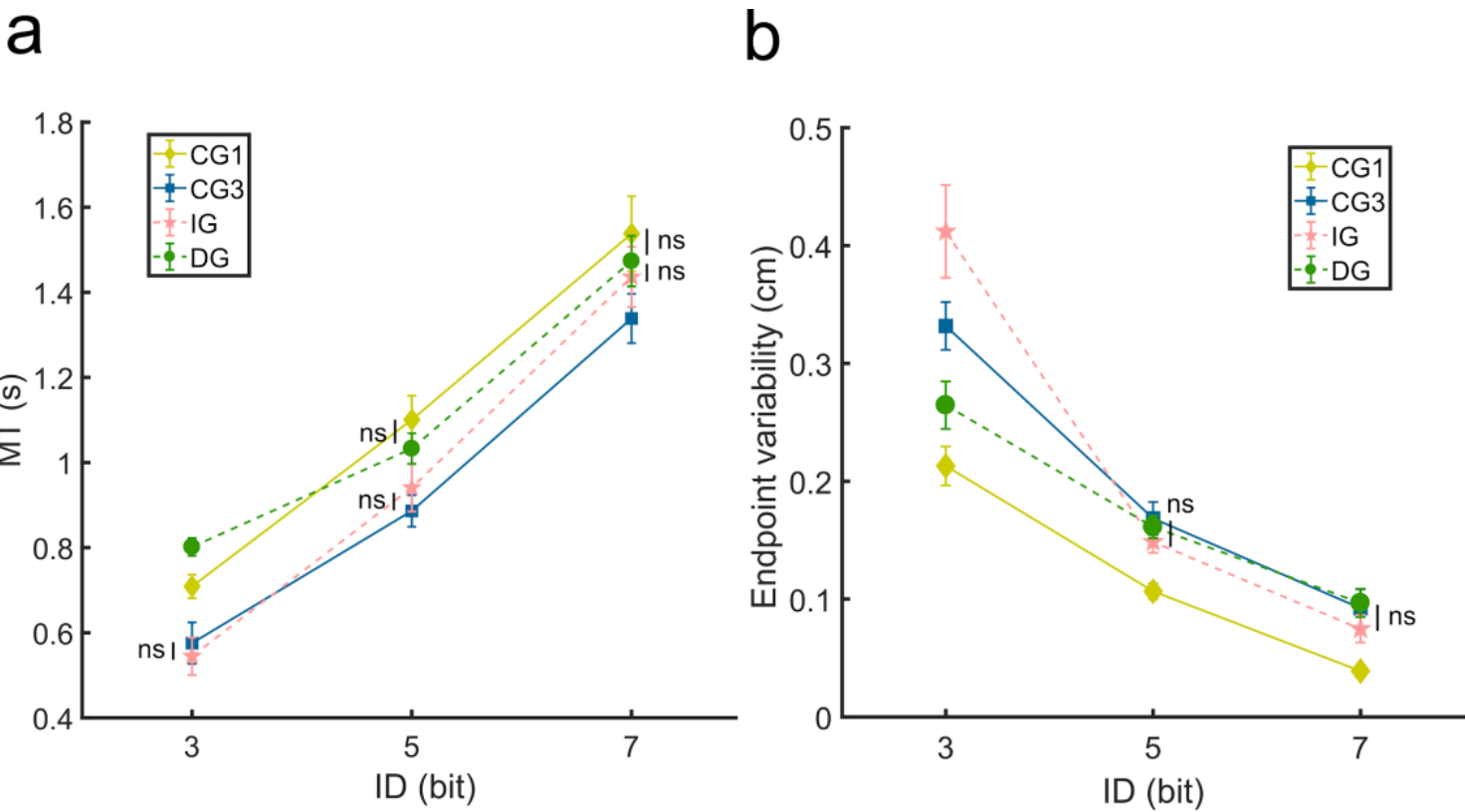

Figure 3: a) Movement Time (MT) and. b) Endpoint variability of the visual cursor in the target versus the Index of Difficulty (ID) and the CD gain. All these comparisons were significant except for those noted $n s$ : non-significant. Error bars denote standard errors.

The pointing performance was also assessed through the endpoint variability: The standard deviation of the visual cursor endpoint in the target. The ANOVA revealed a main effect of ID, CD gain as well as an interaction. The variability decreased with ID because of target width reduction (Fig. 3b). Post-hoc analysis of the interaction showed that CG1 induced the lowest endpoint variability compared to the other CD gains, whatever the ID. The other CD gains were associated with similar endpoint variability at ID5 and ID7. By contrast, when the target was wide (ID3), the endpoint variability was higher for CG3 than DG $(0.33 \pm 0.02 \mathrm{~cm}$ and $0.26 \pm 0.02 \mathrm{~cm}$, respectively; $\mathrm{p}<.01)$ and even higher for IG $(0.41 \pm 0.04 \mathrm{~cm} ; \mathrm{p}<.001)$.

Further analyses were conducted in order to determine what was responsible for the differences observed in the subjects' pointing performance. For this purpose, the kinematics of the movement was analyzed in the motor space through spatial and temporal parameters.

\section{Kinematics}




\section{Peak Velocity}

First, we analyzed how fast the pointing movements of the participants were. The ANOVA performed on the PV in the motor space revealed a main effect of ID , CD Gain as well as an interaction (Table 1; Fig.4a). Therefore, differences in PV between CD gains were linked to the ID: the easier the pointing task the higher the PV. Although the PV at CG1 was significantly greater than the other CD gains regardless of the ID ( $p$ always >.001), this was not the case with DG, which no longer differed from IG at ID7 ( $\mathrm{p}=.16)$. In addition, CG3 and IG were similar at ID3 and started to differ only at ID5 $(\mathrm{p}<.01$ at ID5 and $\mathrm{p}<.05$ at ID7).

\section{Pointing Amplitude}

Secondly, we examined the amplitude of the pointing movements in the various conditions. The movement Amplitude in the motor space (i.e, Amotor) was found to vary drastically between CD gains (Table 1; Fig. 4b). The ANOVA failed to show any effect of ID , which was not surprising as we only manipulated the target width and not the amplitude. However, the analyses revealed a main effect of CD gain as well as an interaction. Obviously, the amplitude produced with the finger was related to the gain at maximum motor velocity. It was therefore similar to the visual amplitude with CG1 (i.e., target amplitude $=8 \mathrm{~cm}$ ), and three times lower with CG3 (Fig. 4b). In addition, the motor amplitude increased relative to ID with IG (i.e., p<.001 in all these comparisons) as the motor velocity decreased (see PV section above), and hence the visual velocity, which required larger pointing movements to be maintained in the motor space. However, the motor amplitude decreased relative to ID with DG (i.e., $\mathrm{p}<.001$ in all these comparisons), and hence the visual velocity increased, which reduced the motor amplitude required to reach the target.

\section{Distance of the first sub-movement}

The distance of the first sub-movement (i.e., rDfirstmotor) corresponds to the motor distance covered at the end of the primary sub-movement. It was computed as a percentage relative to the total motor distance of each pointing movement (i.e., Amotor) as the amplitude required to reach the target varied from one trial to another (except in the case of constant CD gains). This parameter reflected the late on-line corrections carried out: The shorter the rDfirstmotor, the earlier the motor corrections took place. The ANOVA performed on rDfirstmotor revealed a main effect of ID , CD gain as well as an interaction (Table 1; Fig. 4c). Overall, rDfirstmotor decreased as the ID increased. In addition, DG induced the lowest rDfirstmotor compared to the other CD gains at ID3 ( $\mathrm{p}<.001$ in all these comparisons): It therefore induced the earliest corrections. This difference in motor control disappeared with the ID increase as all the CD gains were similar at ID5 and ID7. 

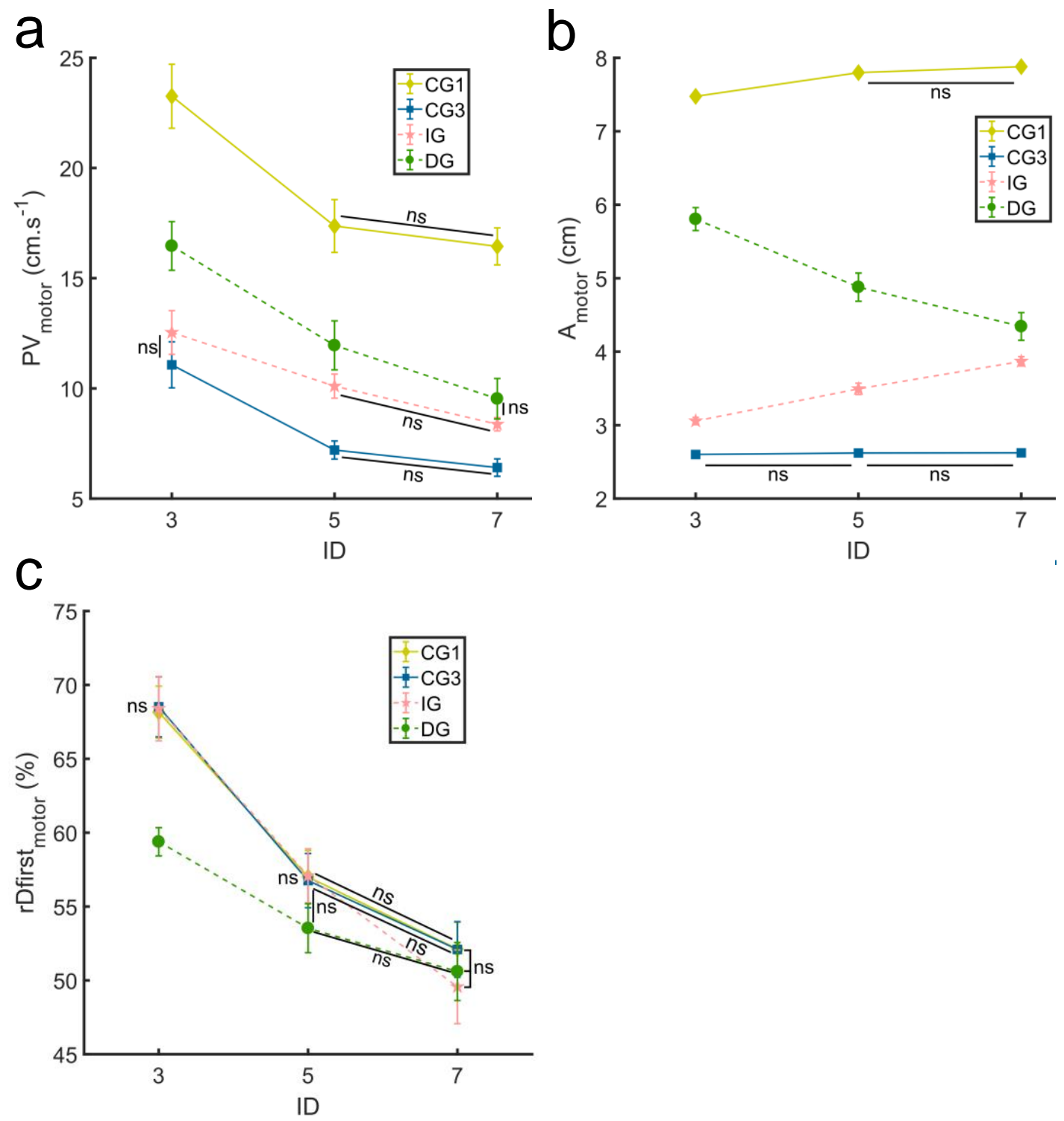

Figure 4: Motor kinematic parameters. a) Peak Velocity in the motor space (PVmotor) versus the Index of Difficulty (ID) and the CD gain. CG1: Constant Gain of 1, CG3: Constant Gain of 3; IG: Increasing Gain; DG: Decreasing Gain. b) Motor amplitude (Amotor) versus the ID and the CD gains. c) Distance at which the primary sub-movement occurred relative to the total motor distance (rDfirstmotor), depending on the ID and the CD gain. All these comparisons were significant except for those noted $n s$ : non-significant. Error bars denote standard errors.

\section{Acceleration time}

The duration of the acceleration time (AT) corresponds to the absolute time elapsing between the movement onset and the PV in the motor space. This parameter is associated with the impulse phase of motor control reflecting the movement planning process. The analyses showed a main effect of ID , and CD gain (Table 1; Fig. 5a) but not for the interaction. Therefore, AT was shorter at ID3 (0.208 \pm $0.008 \mathrm{~s})$ than at ID5 $(0.241 \pm 0.010 \mathrm{~s} ; \mathrm{p}<.01)$ and ID7 $(0.254 \pm 0.009 \mathrm{~s} ; \mathrm{p}<.001)$. Moreover, AT was longer with CG1 and DG compared to IG and CG3 ( $\mathrm{p}<.001$ in all the comparisons between these two groups).

\section{Deceleration time}

The duration of the deceleration time (DT) corresponds to the absolute time elapsing between the PV in the motor space and the end of the movement. This parameter is associated with the correction phase 
reflecting the online movement corrections (e.g., Woodworth 1899; Meyer et al. 1982). The analyses showed an effect of ID , CD gain as well as an interaction (Table 1; Fig. 5b). Overall, these results were similar to those obtained on the MT: At ID3, DG induced the highest DT ( $p<.05$ in the comparison with CG1 and $p<.001$ in the comparisons with CG3 and IG), followed by CG1 ( $<<.05$ in the comparison with CG3 and $\mathrm{p}<.01$ in that with IG) and by CG3 and IG, which were associated with the fastest DT. With the hardest target (ID7), smaller differences in DT were observed: Only CG3 differed from CG1 $(\mathrm{p}<.001), \mathrm{IG}(\mathrm{p}<.01)$ and DG $(\mathrm{p}<.01)$, which included a similar period of motor corrections. Again at ID5, only CG3 differed from CG1 ( $\mathrm{p}<.001)$ and DG ( $\mathrm{p}<.01)$. However, contrary to MT, CG3 induced a shorter DT than IG $(\mathrm{p}<.01)$.
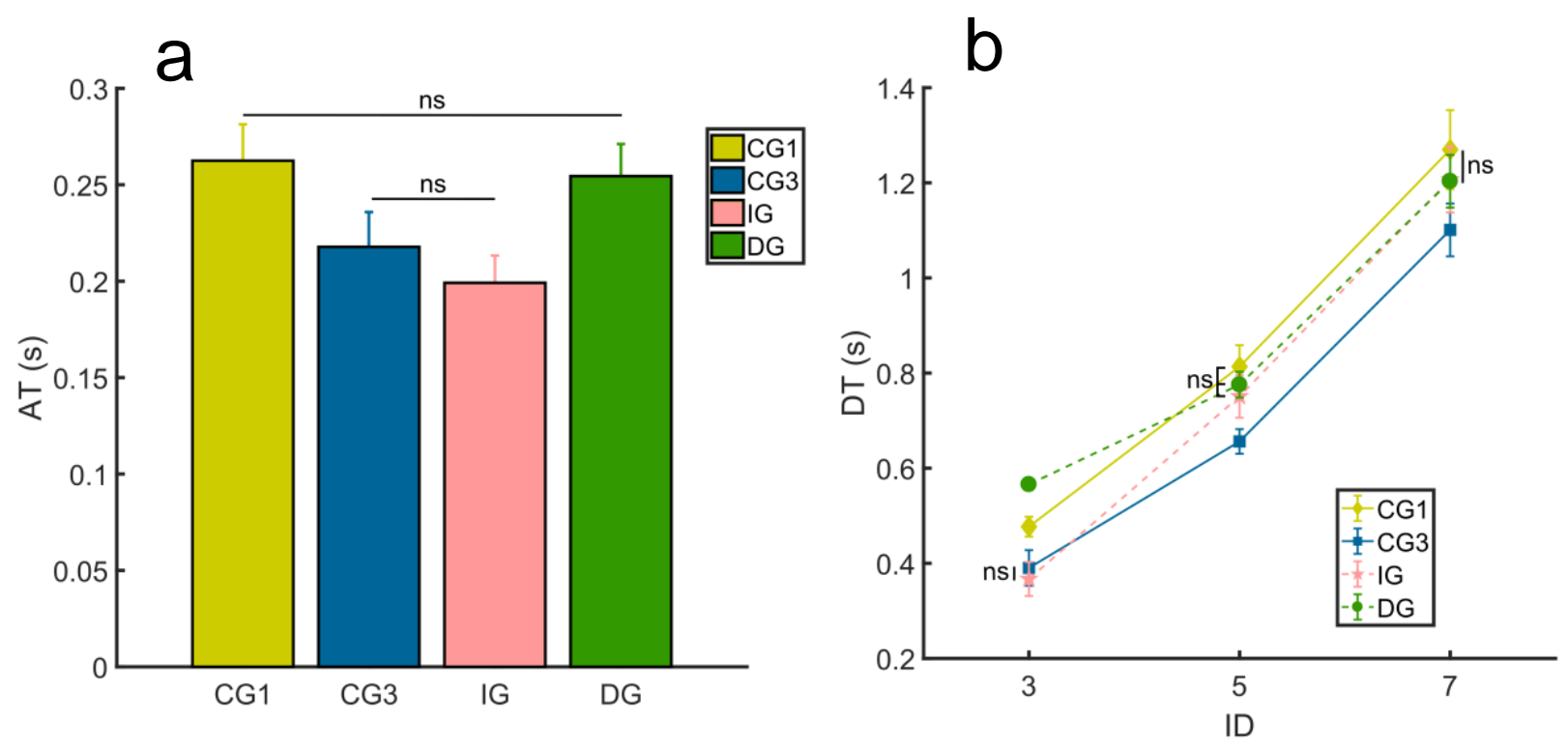

Figure 5: Temporal kinematic parameters. a) Acceleration Time (AT) versus the CD gain. CG1: Constant Gain of 1, CG3: Constant Gain of 3; IG: Increasing Gain; DG: Decreasing Gain. b) Deceleration Time (DT) versus the ID and CD gain. All these comparisons were significant except for those noted $n s$ : non-significant. Error bars denote standard errors.

\section{Discussion}

The aim of the present study was to characterize the motor performance and kinematics of pointing movements with linear (but non-constant) visuomotor gains. This analysis might have important implications in HCI for designing CD gains enhancing users' performance on indirect interfaces. Although the $\mathrm{CD}$ gains used on modern OSs have been found to improve pointing performance (Casiez et al. 2008), the reasons for this improvement have not yet been completely elucidated, mainly because these OS gains are usually both non-constant and non-linear. Here we tested non-constant but linear velocity-based $\mathrm{CD}$ gains with a view to determining which parameters were responsible for the differences in subjects' pointing performance. Constant CD gains of 1 (CG1) and 3 (CG3) were compared with a linearly Increasing CD Gain (IG) and a linearly Decreasing CD Gain (DG). We first observed that whatever the CD gain tested, MT increased linearly with the ID, as found to occur with the non-constant non-linear CD gain associated with computer OSs (i.e., Windows XP; Casiez et al. 2008). In the present study, Fitts' law still held with visuomotor mappings which were non-constant and linear and even with a Decreasing Gain. Overall, CD gain was found to have a greater impact at low IDs, and a more stereotyped movement profile was observed with all the $\mathrm{CD}$ gains tested at the highest ID (i.e., smaller differences between CD gains were observed at ID7 in the case of MT, DT and 
rDfirstmotor). In addition, similarities in the motor planning, investigated through AT, between CG1 and DG on one hand, and CG3 and IG on the other hand. Contrary to our expectations, we did not find any clear-cut improvements in the pointing performance with IG or any clear-cut deterioration with DG. IG was initially expected to increase the velocity of the initial impulse phase and decrease the velocity of the correction phase, thus improving the pointing performance; and the contrary in the case of DG.

DG might have been expected to have a strong impact on the pointing performance since it reverses the link between motor and visual velocity: The faster the finger moves, the slower the visual cursor moves. This CD gain did not slow down the pointing movement drastically, especially in comparison with CG1. The greatest impact of DG was found to occur at ID3 because it required participants to have a high motor velocity: With DG, reaching a visual velocity greater than $11.25 \mathrm{~cm}$.s1 required a velocity of more than $11.25 \mathrm{~cm} . \mathrm{s}-1$ in the motor space (see Figure 1c). A non-increasing relationship was found to exist between the motor and visual velocities. Up to a motor velocity of 7.5 cm.s-1, the visual velocity increased before decreasing at a motor velocity ranging from 7.5 to $10 \mathrm{~cm}$.s1. As a consequence, the endpoint variability in the visual target was higher than CG1. This poor performance achieved with DG in comparison with CG1 seemed to be due to the motor PV being too low and the correction phase (i.e., DT) being too long. Indeed, both CD gains showed similar ATs, while the DTs clearly differed. The time required to reach PV was therefore not responsible for poor performance. More specifically, DG was found to be associated with a shorter rDfirst. The first submovement involved in DG was equal to 59\% of the total motor amplitude, whereas it amounted to 68\% in the case of CG1. DG was therefore associated with longer movement correction distances reflecting the occurrence of early corrections (Elliott et al. 2010). During the subsequent sub-movement(s), sensory cues (visual and kinesthetic cues) are picked up about the limb position and the target, and movement corrections are triggered (Woodworth 1899; Meyer et al. 1982; Elliott et al. 2001). Overall, whatever the value of the $\mathrm{CD}$ gain, the higher the ID, the larger the late corrections, which suggests the general need for sensory cues processing in order to achieve the task (Elliott et al. 1999).

One might have expected CG3 to have a negative effect on the pointing performance with high IDs due to the decrease in the target size occurring in the motor space. A CD gain of 3 could be considered as detrimental to the performance as fine motor adjustments were not possible in the case of CG3. This was not the case here, however. Besides, CG3 was even associated with the highest performance in the hardest pointing task (i.e., with the shortest MTs at ID7). The time saved during the impulse phase (i.e., AT shortening) probably explained why CG3 was still associated with better performance than CG1 despite its high sensitivity. Casiez et al. (2008) have previously described this $L$ shaped performance profile upon comparing constant CD gains. A fast MT decrease occurred with increasing CD gains but little degradation of the performance was observed at high CD gains, except with very small targets. Contrary to our predictions, IG was not associated with better performance than CG3. At ID7, MT was even higher with IG than CG3, and at ID3, IG induced more endpoint variability in the target than CG3. As the initial impulse phase needs to be performed at high speed and the following correction phase needs to be performed at low speed (Woodworth 1899; Meyer et al. 1982; Elliott et al. 2001), the IG function could have been more appropriated. This result could have been due to the decrease in the motor PV observed with increasing IDs. The movement amplitude required would therefore have increased, as well as the duration of the correction phase (i.e., with longer DTs in the case of IG than in that of CG3 at ID5 and ID7).

The non-constant visuomotor mapping of IG, as well as that of DG, suggests that the participants had difficulty in controlling the pointing movement efficiently. In both conditions, the performance decreased compared to the corresponding constant gain (i.e., CG3 and CG1, respectively). IG was therefore associated with longer MTs than CG3 at ID7. In addition, DG was associated with longer MTs at ID3 than all the other CD gains. Several studies have previously shown that changing the gain between 
a motion and its consequences in the visual space can disrupt the sensorimotor control (Ferrel et al. 2000; Klatzky et al. 2003; Coello et al. 2004; Bohan et al. 2010). Performance would not improve when the visuomotor mapping is too complex to be understood and efficiently used by the SNC. Ferrel et al. (2000) have established in addition that the quality of the adaptation is poor and incomplete when the constant CD gain is randomized across trials. On similar lines, the non-constant gains occurring in the present study (i.e., DG and IG) might be taken to have changed with time and/or from one trial to another, thus limiting the improvement of the performance. The participants may not have determined the relationship between the visual and motor spaces and assumed the visuomotor mapping random.

Movement adaptations rely on preliminary motor commands based on predictions and on-line adjustments (made during the execution phase) and off-line adjustments (made during subsequent trials) based on multisensory feedback (Blouin et al. 1993; Khan et al. 2003). This updating of pointing movements requires mainly visual processing of sensory information (see Sarlegna and Mutha 2015) as well as proprioceptive processing (Sainburg et al. 1995; Scott 2012). Vision has been found to be particularly crucial during the correction phase, when the effector is approaching the target (Desmurget et al. 1995). In addition, studies in which constant CD gains were manipulated showed that the discrepancies were higher in the late phase of the movement (Elliott et al. 1997; Ferrel et al. 2000; van Doorn et al. 2005), when visual cues play a major role. Here we extended this finding to include visuomotor mappings which were linear and non-constant, as the greatest influence of CD gain was found to be exerted on the DT associated with the correction phase. However, the present results suggest that the motor commands were at least partly tuned relative to the visuomotor mapping. For instance, we observed a clear modification of PV in the motor space relative to the CD gains, which suggested that the specificity of the visuomotor mapping was taken into account early in the course of the trial. This processing may not have been optimal, resulting in disruptions in the sensorimotor control (Ferrel et al. 2000; Klatzky et al. 2003; Coello et al. 2004; Bohan et al. 2010), as mentioned above. In addition, with the parametrization of the motor commands in the case of a specific visuomotor mapping, the temporal organization of the motor kinematics seemed to be associated to commonalties. Therefore, the AT, reflecting the duration of the impulse phase, was similar between CG1 and DG on one hand, and between CG3 and IG on the other hand. In addition, the fact that a similar motor PV was observed between CG1 and DG and between CG3 and IG suggests that the visuomotor mapping at the maximum motor velocity tuned, at least partly, how the brain programmed the action. More specifically, the motion profile might be based on this gain observed at the maximum motor velocity, and the amplitude of the curve (i.e., PV) might be specific to each visuomotor mapping.

Further studies would be necessary to confirm these hypotheses and to characterize the impact of visuomotor mapping on sensorimotor kinematics in general. This topic of research has several potential applications, which mainly involve to the use of indirect interfaces (e.g., laptops, telesurgery). Understanding and optimizing users' performance via computer CD gains constitutes one of the key issues at stake in the field of HCI. It was established here in particular that an increasing gain was not necessarily associated with an improvement in subjects' performance using a trackpad. This conclusion still remains to be confirmed with other IG functions with different shapes and maximum gains, and should also be extended to the use of a mouse. However, it suggests that a low gain at low velocity does not necessarily speed up the movement. This is consistent with empirical findings on CD gains which have been implemented in operating systems. For instance, with Windows 10 and OSX (associated with a mouse), the CD gains are non-constant and non-linear with a logarithmic relationship at low motor speeds at which the visuomotor gain increases drastically. Casiez and Roussel (2011) already showed shorter pointing movements with these operating systems in comparison with a constant CD gain of 1.5. First, this result needs to be confirmed by making comparisons with higher constant CD gains known to 
decrease the MT (Accot and Zhai 2001; Kovacs et al. 2008; Bohan et al. 2010). Secondly, we think that the ideal CD gain should not be based on motor velocity as it has been up to now, but on the motor acceleration parameter. With such visuomotor mapping, the gain will be high during the whole acceleration phase (i.e., the impulse phase) and low during the deceleration phase (i.e., during the correction phase), and is liable to improve the subjects' performance.

\section{References}

Accot J, Zhai S (2001) Scale effects in steering law tasks. In: Proceedings of the SIGCHI conference on Human factors in computing systems - CHI '01. ACM Press, Seattle, Washington, United States, pp 1-8

Balakrishnan R (2004) “Beating” Fitts' law: virtual enhancements for pointing facilitation. Int J Hum-Comput Stud 61:857-874. https://doi.org/10.1016/j.ijhcs.2004.09.002

Blanch R, Guiard Y, Beaudouin-Lafon M (2004) Semantic Pointing: Improving Target Acquisition with Control-display Ratio Adaptation. In: Proceedings of the SIGCHI Conference on Human Factors in Computing Systems. ACM, New York, NY, USA, pp 519-526

Blouin J, Bard C, Teasdale N, Fleury M (1993) On-Line versus Off-Line Control of Rapid Aiming Movements. J Mot Behav 25:275-279. https://doi.org/10.1080/00222895.1993.9941648

Bohan M, McConnell DS, Chaparro A, Thompson SG (2010) The effects of visual magnification and physical movement scale on the manipulation of a tool with indirect vision. J Exp Psychol Appl 16:33-44. https://doi.org/10.1037/a0018501

Boyle J, Kennedy D, Shea CH (2012) Optimizing the control of high ID movements: rethinking the obvious. Exp Brain Res 223:377-387. https://doi.org/10.1007/s00221-012-3266-6

Brenner E, Smeets JBJ (2011) Quickly 'learning' to move optimally. Exp Brain Res 213:153161. https://doi.org/10.1007/s00221-011-2786-9

Card SK, English WK, Burr BJ (1978) Evaluation of Mouse, Rate-Controlled Isometric Joystick, Step Keys, and Text Keys for Text Selection on a CRT. Ergonomics 21:601-613. https://doi.org/10.1080/00140137808931762

Casiez G, Roussel N (2011) No more Bricolage! Methods and Tools to Characterize, Replicate and Compare Pointing Transfer Functions. ACM Press, New York, USA, pp 603-614

Casiez G, Roussel N, Vanbelleghem R, Giraud F (2011) Surfpad: riding towards targets on a squeeze film effect. In: Proceedings of the 2011 annual conference on Human factors in computing systems - CHI '11. ACM Press, Vancouver, BC, Canada, p 2491

Casiez G, Vogel D, Balakrishnan R, Cockburn A (2008) The Impact of Control-Display Gain on User Performance in Pointing Tasks. Hum-Comput Interact 23:215-250. https://doi.org/10.1080/07370020802278163

Coello Y, Milleville-Pennel I, Orliaguet JP (2004) Position coding in a video-controlled pointing task with a rotated visual display: evidence for individual differences in visuo-proprioceptive interaction. Neurosci Lett 369:214-218. https://doi.org/10.1016/j.neulet.2004.07.066 
Desmurget M, Rossetti Y, Prablanc C, et al (1995) Representation of hand position prior to movement and motor variability. Can J Physiol Pharmacol 73:262-272. https://doi.org/10.1139/y95037

Elliott D, Binsted G, Heath M (1999) The control of goal-directed limb movements: Correcting errors in the trajectory. Hum Mov Sci 18:121-136. https://doi.org/10.1016/S0167-9457(99)00004-4

Elliott D, Hansen S, Grierson LEM, et al (2010) Goal-directed aiming: Two components but multiple processes. Psychol Bull 136:1023-1044. https://doi.org/10.1037/a0020958

Elliott D, Helsen WF, Chua R (2001) A century later: Woodworth's (1899) two-component model of goal-directed aiming. Psychol Bull 127:342-357

Elliott D, Lyons J, Dyson K (1997) Rescaling an acquired discrete aiming movement: Specific or general motor learning? Hum Mov Sci 16:81-96. https://doi.org/10.1016/S0167-9457(96)00041-3

Fernandez L, Bootsma RJ (2008) Non-linear gaining in precision aiming: Making Fitts' task a bit easier. Acta Psychol (Amst) 129:217-227. https://doi.org/10.1016/j.actpsy.2008.06.001

Ferrel C, Leifflen D, Orliaguet J-P, Coello Y (2000) Pointing movement visually controlled through a video display: adaptation to scale change. Ergonomics 43:461-473. https://doi.org/10.1080/001401300184341

Fitts PM (1954) The information capacity of the human motor system in controlling the amplitude of movement. J Exp Psychol 47:381-391. https://doi.org/10.1037/h0055392

Fradet L, Lee G, Dounskaia N (2008) Origins of submovements during pointing movements. Acta Psychol (Amst) 129:91-100. https://doi.org/10.1016/j.actpsy.2008.04.009

Khan MA, Lawrence G, Fourkas A, et al (2003) Online versus offline processing of visual feedback in the control of movement amplitude. Acta Psychol (Amst) 113:83-97. https://doi.org/10.1016/s0001-6918(02)00156-7

Klatzky RL, Lederman SJ, Langseth S (2003) Watching a Cursor Distorts Haptically Guided Reproduction of Mouse Movement. J Exp Psychol Appl 9:228-235. https://doi.org/10.1037/1076898X.9.4.228

Kovacs AJ, Buchanan JJ, Shea CH (2008) Perceptual influences on Fitts' law. Exp Brain Res 190:99-103. https://doi.org/10.1007/s00221-008-1497-3

Krakauer JW, Pine ZM, Ghilardi M-F, Ghez C (2000) Learning of Visuomotor Transformations for Vectorial Planning of Reaching Trajectories. J Neurosci 20:8916-8924. https://doi.org/10.1523/JNEUROSCI.20-23-08916.2000

Levesque V, Oram L, MacLean K, et al (2011) Enhancing physicality in touch interaction with programmable friction. In: Proceedings of the 2011 annual conference on Human factors in computing systems - CHI '11. ACM Press, Vancouver, BC, Canada, p 2481

MacKenzie CL, Marteniuk RG, Dugas C, et al (1987) Three-Dimensional Movement Trajectories in Fitts' Task: Implications for Control. Q J Exp Psychol Sect A 39:629-647. https://doi.org/10.1080/14640748708401806 
MacKenzie IS (1992) Fitts' Law as a Research and Design Tool in Human-Computer Interaction. Human-Computer Interact 7:91-139. https://doi.org/10.1207/s15327051hci0701_3

Meyer DE, Smith JE, Wright CE (1982) Models for the speed and accuracy of aimed movements. Psychol Rev 89:449-482

Rossetti Y, Koga K, Mano T (1993) Prismatic displacement of vision induces transient changes in the timing of eye-hand coordination. Percept Psychophys 54:355-364

Sainburg RL, Ghilardi MF, Poizner H, Ghez C (1995) Control of limb dynamics in normal subjects and patients without proprioception. J Neurophysiol 73:820-835. https://doi.org/10.1152/jn.1995.73.2.820

Sarlegna FR, Mutha PK (2015) The influence of visual target information on the online control of movements. Vision Res 110:144-154. https://doi.org/10.1016/j.visres.2014.07.001

Scott SH (2012) The computational and neural basis of voluntary motor control and planning. Trends Cogn Sci 16:541-549. https://doi.org/10.1016/j.tics.2012.09.008

Seidler RD, Bloomberg JJ, Stelmach GE (2001) Context-dependent arm pointing adaptation. Behav Brain Res 119:155-166. https://doi.org/10.1016/s0166-4328(00)00347-8

van Doorn RRA, Unema PJA, Hendriks EJ (2005) The locus of adaptation to altered gain in aimed movements. Hum Mov Sci 24:31-53. https://doi.org/10.1016/j.humov.2005.03.001

Woodworth RS (1899) Accuracy of voluntary movement. Psychol Rev Monogr Suppl 3:i-114. https://doi.org/10.1037/h0092992 